\title{
Minerais e amido resistente em farinhas de banana vermelha 'São Domingos' triploide (AAA)
}

\author{
Minerals and resistant starch in red banana flours 'São Domingos' triploid (AAA) \\ Minerales y almidón resistente en harinas de plátano rojo triploide 'São Domingos' (AAA)
}

Recebido: 13/03/2021 | Revisado: 20/03/2021 | Aceito: 21/03/2021 | Publicado: 28/03/2021

\author{
Hebert Teixeira Cândido \\ ORCID: https://orcid.org/0000-0002-3040-6488 \\ Universidade Estadual Paulista, Brasil \\ E-mail: hebert.candido@unesp.br \\ Nicholas Zanette Molha \\ ORCID: https://orcid.org/0000-0002-4866-4258 \\ Universidade Estadual Paulista, Brasil \\ E-mail: nz.molha@unesp.br \\ Juliana Aparecida Marques Eburneo \\ ORCID: https://orcid.org/0000-0002-7522-6405 \\ Universidade Estadual Paulista, Brasil \\ E-mail: julianaeburneo@gmail.com \\ Magali Leonel \\ ORCID: https://orcid.org/0000-0001-7896-2398 \\ Universidade Estadual Paulista, Brasil \\ E-mail: magali.leonel@unesp.br
}

\begin{abstract}
Resumo
A banana é uma das principais culturas agrícolas do mundo, de grande importância socioeconômica para os países em via de desenvolvimento e com características importantes para a fabricação de farinhas. Dente as variedades de bananas, existem aquelas de cascas vermelhas, as quais se destacam pelos altos teores de compostos bioativos, como, por exemplo, precursores da vitamina A. Contudo, ainda existem lacunas no conhecimento em relação ao amido resistente e minerais presentes nessas variedades. Assim, o estudo teve como objetivo caracterizar os teores de minerais e amido resistente presentes nas farinhas de casca e de polpa da banana de casca vermelha 'São Domingos' (AAA). As farinhas, casca e polpa, foram produzidas a partir de frutos obtidos em uma propriedade rural, sanitizados, fatiados, descascados, secos em estufa com circulação de ar forçada a $55^{\circ} \mathrm{C}$ e moídos em moinho rolo-facas. Dessa forma, foram submetidas às análises de umidade, cinzas, minerais (potássio, fósforo, cálcio, magnésio, enxofre, cobre, manganês, zinco, ferro e sódio) e amido resistente. As farinhas não diferiram no teor de umidade. A farinha de casca apresentou maiores teores de cinzas, potássio, magnésio, fósforo, zinco e sódio. Potássio e manganês foram os, macro e micromineral, presentes em maiores quantidades em ambas as farinhas. Ambas as farinhas apresentaram umidade dentro do limite estabelecido pela legislação. A farinha de casca possui maior teor de minerais; enquanto a farinha de polpa possui quase o dobro de amido resistente. As farinhas de banana de casca vermelha 'São Domingos' possuem características semelhantes às de outras triploides AAA.
\end{abstract}

Palavras-chave: Musa spp; Farinha de polpa de banana verde; Farinha de casca de banana verde.

\begin{abstract}
Banana is one of the main agricultural crops in the world, of great socioeconomic importance for developing countries and with important characteristics for the manufacture of flour. Among the varieties of bananas, there are those with red peels, which stand out for their high levels of bioactive compounds, such as precursors of vitamin A. However, there are still gaps in knowledge regarding resistant starch and minerals present in these varieties. Thus, the study aimed to characterize the levels of minerals and resistant starch present in the flour of the peel and pulp of the red banana 'São Domingos' (AAA). The flours, peel and pulp, were produced from fruits obtained in a rural property, sanitized, sliced, peeled, dried in an oven with forced air circulation at $55^{\circ} \mathrm{C}$ and ground in a roller-knife mill. Thus, they were subjected to the analysis of moisture, ash, minerals (potassium, phosphorus, calcium, magnesium, sulfur, copper, manganese, zinc, iron and sodium) and resistant starch. Flours did not differ in moisture content. The bark flour showed higher levels of ash, potassium, magnesium, phosphorus, zinc and sodium. Potassium and manganese were the, macro and micromineral, present in greater quantities in both flours. Both flours showed moisture within the limit established by the legislation. Shell flour has a higher mineral content; while pulp flour has almost twice as much resistant starch. The 'São Domingos' red-skinned banana flours have characteristics similar to those of other AAA triploids.
\end{abstract}

Keywords: Musa spp; Unripe banana pulp flour; Unripe banana peel flour. 


\begin{abstract}
Resumen
El banano es uno de los principales cultivos agrícolas del mundo, de gran importancia socioeconómica para los países en desarrollo y con características importantes para la fabricación de harinas. Entre las variedades de banano, se encuentran las de piel roja, que destacan por los altos niveles de compuestos bioactivos, como, por ejemplo, los precursores de la vitamina A. Sin embargo, aún existen lagunas en el conocimiento sobre el almidón resistente y los minerales presentes en estas variedades. Así, el estudio tuvo como objetivo caracterizar los niveles de minerales y almidón resistente presentes en las harinas de cáscara y pulpa del plátano rojo 'São Domingos' (AAA). Las harinas, cáscara y pulpa, se elaboraron a partir de frutos obtenidos en una finca local, higienizados, cortados, pelados, secados en horno con circulación de aire forzado a $55^{\circ} \mathrm{C}$ y molidos en molino de cuchillas. Así, fueron sometidos al análisis de humedad, cenizas, minerales (potasio, fósforo, calcio, magnesio, azufre, cobre, manganeso, zinc, hierro y sodio) y almidón resistente. Las harinas no difirieron en contenido de humedad. La harina de cáscara mostró niveles más altos de ceniza, potasio, magnesio, fósforo, zinc y sodio. El potasio y el manganeso fueron los macro y microminerales, presentes en mayor cantidad en ambas harinas. Ambas harinas presentaron humedad dentro del límite establecido por la legislación. La harina de concha tiene un mayor contenido de minerales; mientras que la harina de pulpa tiene casi el doble de almidón resistente. Las harinas del plátano rojo 'São Domingos' tienen características similares a las de otros triploides AAA.
\end{abstract}

Palabras clave: Musa spp; Harina de pulpa de plátano verde; Harina de cáscara de plátano verde.

\title{
1. Introdução
}

A banana é uma das frutas mais consumidas no mundo, cultivada principalmente em zonas tropicais e subtropicais de países em via de desenvolvimento. Dentre os principais produtores, destacam-se a Índia, China, Indonésia, Equador, Angola, Costa Rica, Tanzânia, dentre outros grandes produtores, de modo que o Brasil aparece em quarto lugar com 6.812.708 toneladas produzidas anualmente (Food and Agriculture Organization of the United Nations: FAOSTAT [FAO], 2021). No cenário Brasileiro, a cultura é explorada em todas as unidades da federação e, dentre as culturas permanentes, àquela cultivada em maior número de estabelecimentos rurais, com valor de produção anual superior à 3,7 bilhões de reais (Instituto Brasileiro de Geografia e Estatística [IBGE], 2021).

Existem muitos cultivares de bananas difundidos no Brasil, principalmente dos subgrupos: Terra, Maçã, Cavendish e Prata (Amorim et al., 2016). Entretanto, outros cultivares menos difundidos também possuem características nutricionais, sensoriais e culturais de potencial econômico. Dentre esses cultivares, tem-se a banana 'São Domingos' (Musa X paradisíaca), uma triploide AAA de casca vermelha (Lorenzi et al., 2015), a qual possui características físico-químicas semelhantes às de outras bananas AAA (Souza et al., 2019). O tom avermelhado das cascas dessas bananas pode ser um atrativo para o mercado (Lekshmi et al., 2019). Além disso, bananas vermelhas possuem altos níveis dos carotenoides: luteína, $\beta$-caroteno e $\alpha$-caroteno e de antocianinas, sendo essas últimas, as principais responsáveis pela coloração avermelhada de suas cascas (Fu et al., 2018). Os teores de flavonoides, antocianinas e carotenoides são maiores em casca do que na polpa (Fu et al., 2018). No entanto, devido à presença de carotenoides pró-vitamina $\mathrm{A}$, a polpa também tem potencial para auxiliar na deficiência dessa vitamina (Lokesh et al., 2014), a qual apresenta deficiência de ingestão em algumas regiões brasileiras (Cozzolino, 2007).

Os frutos de bananas verdes podem ser utilizados para a produção de farinhas, sejam elas fabricadas a partir da polpa, da casca, ou integral (casca e polpa sem separação) as quais podem sem empregadas para uma ampla gama de produtos panificados. Assim, estudos têm mostrado viabilidade na fabricação de pães preparados com farinhas de banana verde (polpa, casca ou integral) em substituição parcial à farinha de trigo. De modo que os produtos obtidos têm apresentado boa aceitação e ganhos nas qualidades tecnológicas e nutricionais, principalmente nos teores de minerais, fibras e amido resistente (Al-Sahlany \& Al-musafer, 2020; Alcântara et al., 2020; Khoozani et al., 2020; Silva et al., 2015; Viana et al. 2018). Uma das principais características das farinhas de bananas verdes é o alto teor de amido resistente, o qual proporciona baixo índice glicêmico (Kumar et al., 2019). Isso acontece, pois, esse amido não é digerido no trato digestivo, sendo hidrolisado somente no intestino grosso. Outra vantagem do amido resistente é a de menor interferência nas características sensoriais do produto (Falcomer et al., 2019). 
Os minerais são importantes para a alimentação humana pois estão presentes como componentes de tecidos, síntese de hormônios, sistema imunológico, reguladores de processos metabólicos e funcionamento de órgãos (Gharibzahedi \& Jafari, 2017). Assim, o teor de minerais é uma propriedade importante dos alimentos, visto que atualmente, a ingestão de alimentos, principalmente pelas populações carentes, é majoritariamente por alimentos altamente calóricos e com poucos minerais, causando em muitas ocasiões a fome oculta, principalmente nos países em via de desenvolvimento (Valença et al., 2017). Estudos no Brasil mostram que ferro, cálcio e zinco são os minerais que aparecem com maior frequência de ingestão inferior à recomendada (Cozzolino, 2007). As deficiências de cálcio e zinco, por exemplo, podem levar a um maior acúmulo de gordura corporal (cálcio), retardo no crescimento, obesidade e, em crianças, comprometimento da capacidade cognitiva (zinco) (Leão \& Santos, 2012). Além da anemia causada pela deficiência de ferro (Cozzolino, 2007). Atualmente, mesmo com a grande diversidade de produtos alimentícios disponíveis para o consumidor, o consumo de porções diárias de frutas pode auxiliar no suprimento de minerais (Marles et al., 2017), o qual, em bananas, pode ter o seu teor aumentado em mais de 700\% (casca) e quase 300\% (polpa) após a secagem em estufa (Cândido, 2020).

Dessa forma, o trabalho objetivou comparar os teores de umidades, cinzas, amido resistente e dos minerais: fósforo $(\mathrm{P})$, potássio $(\mathrm{K})$, cálcio $(\mathrm{Ca})$, magnésio $(\mathrm{Mg})$, enxofre $(\mathrm{S})$, ferro $(\mathrm{Fe})$, cobre $(\mathrm{Cu})$, zinco $(\mathrm{Zn})$, manganês $(\mathrm{Mn})$ e sódio $(\mathrm{Na})$ presentes nas farinhas de casca e polpa produzidas com bananas verdes 'São Domingos' (AAA).

\section{Metodologia}

Os frutos foram obtidos em maio de 2019 em uma propriedade rural localizada no município de Botucatu - SP. Para o preparo das farinhas, os frutos foram higienizados em água potável corrente e cortados transversalmente em fatias de aproximadamente $0,5 \mathrm{~cm}$, foram amostrados frutos de todas as pencas, totalizando ao menos 12 frutos por cacho. Após o corte dos frutos, as cascas foram separadas das polpas e, assim, cascas e polpas foram alocadas em bandejas separadas e encaminhadas para secagem em secadores com circulação de ar forçada. A temperatura de secagem foi ajustada em $50{ }^{\circ} \mathrm{C}$, onde o material permaneceu até a estabilização do peso (Al-Sahlany \& Al-musafer, 2020; Viana et al., 2018). A moagem do material foi realizada em moinho de rolo-facas. Dessa forma, obtiveram-se dois produtos: farinha de casca e farinha de polpa.

A umidade das farinhas foi determinada a partir da diferença entre a massa inicial e a massa final, obtida após a secagem em estufa com circulação de ar força a $105^{\circ} \mathrm{C}$ por um período de pelo menos $8 \mathrm{~h}$. $\mathrm{O}$ teor de cinzas foi obtido pela diferença de massa após a passagem pela mufla a $550^{\circ} \mathrm{C}$, conforme metodologia 08-12.01 (American Association of Cereal Chemists [AACC], 1983). Para a quantificação de minerais, as farinhas foram submetidas à digestão com ácido nitroperclórico e encaminhadas para um laboratório para a leitura dos seus teores.

O teor de amido resistente foi obtido seguindo a metodologia proposta por Goñi et al. (1996), que ocorreu por meio de processo enzimático, onde o teor final de amido resistente foi calculado pela concentração da glicose liberada, a qual foi obtida a partir da absorbância em espectrofotômetro a $505 \mathrm{~nm}$.

A análise estatística foi realizada por meio do software SISVAR (Ferreira, 2014), onde para a comparação entre as farinhas de casca e polpa os resultados foram submetidos a análise de variância e teste $\mathrm{F}$ e para a comparação entre os minerais presentes em uma mesma farinha foi utilizado o teste de média Scott-Knott (Vieira, 2006). Todas as análises foram realizadas em triplicata. 
Figura 1. Frutos de banana vermelha var. 'São Domingos' (AAA) utilizados para o preparo das farinhas.

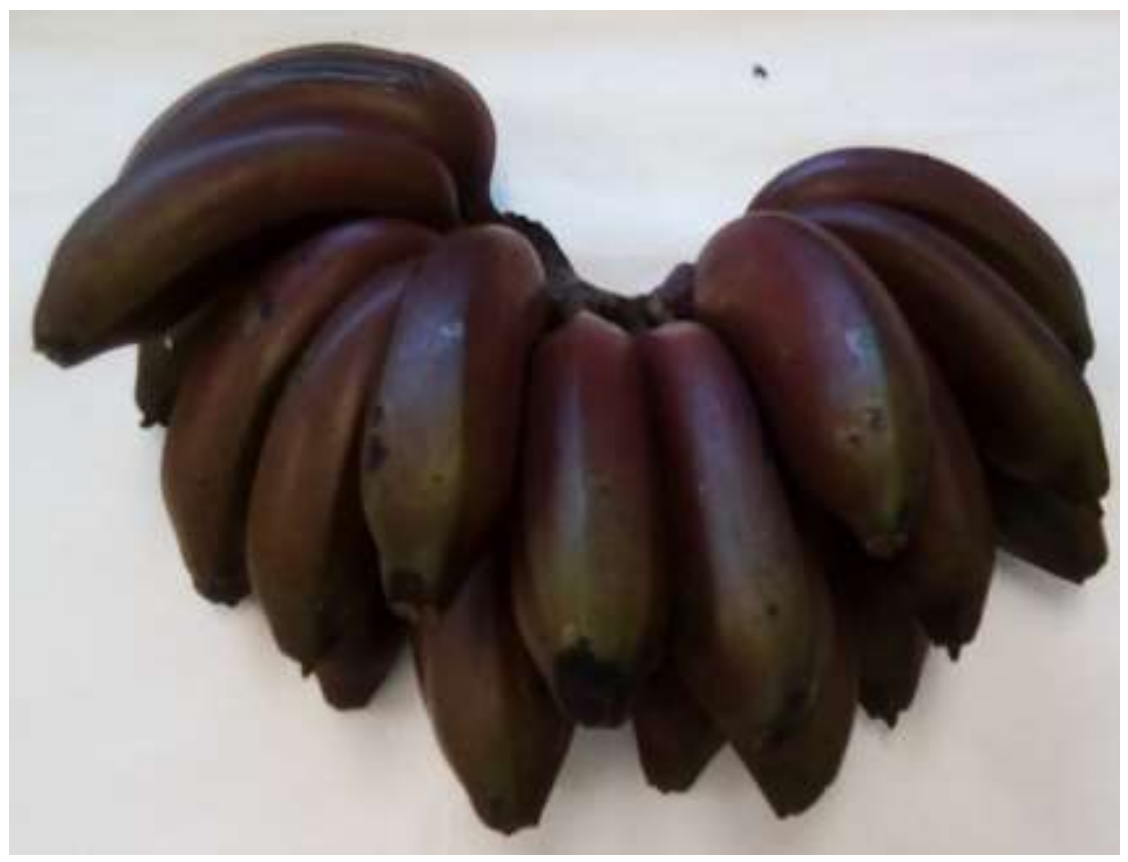

Fonte: Autores.

\section{Resultados e Discussão}

A umidade das farinhas não apresentou diferença estatística ( $p<0,05$; C.V. 2,1\%). Os valores de umidade obtidos

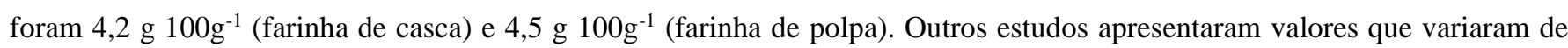
3,3-6,7 g 100 $\mathrm{g}^{-1} \mathrm{em}$ farinha de polpa (Borges et al., 2009; Cândido, 2020; Viana et al., 2018) e de 5,4-7,2 $\mathrm{g} \mathrm{100g}^{-1} \mathrm{em}$ farinha de casca (Cândido, 2020; Eshak, 2016; Sardá et al., 2016). Ambas as farinhas atendem os requisitos de umidade da legislação pertinente (Resolução da Diretoria Colegiada [RDC] n. 263, 2005).

O teor de cinzas das farinhas foi significativamente diferente ( $p<0,05$; C.V. $1,0 \%$ ), apresentando-se em $9,2{\mathrm{~g} 100 \mathrm{~g}^{-1}}^{-1}$

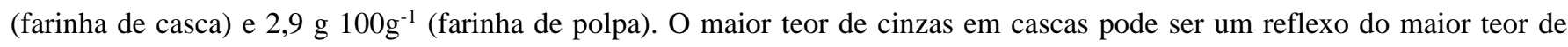
fibras presentes nesse material (Cândido, 2020), visto que os minerais podem estar ligados aos seus componentes (Cozzolino, 1997). No entanto, conforme apresentado por outros estudos, ambas as farinhas de bananas verdes (casca, polpa e integral 'casca e polpa') podem aumentar o teor de cinzas em produtos panificados quando utilizadas em substituição a farinha de trigo (Al-Sahlany \& Al-musafer, 2020; Alcântara et al., 2020; Khoozani et al., 2020; Viana et al., 2018).

Assim como apresentado em estudos com outros cultivares de banana, o mineral mais abundante na farinha de polpa foi o potássio (Tabela 1) (Anyasi et al., 2018; Cândido, 2020; Suntharalingam \& Ravindran, 1993). O cálcio foi, a exceção do sódio, o macromineral presente em menor quantidade em farinhas de polpa (Tabela 1), corroborando outros estudos (Cândido, 2020; Suntharalingam \& Ravindran, 1993).

O potássio também foi o mineral de maior presença na farinha de casca (Tabela 1), concordando com outros estudos (Cândido, 2020; Eshak, 2016). O alto teor de potássio encontrado nas farinhas de casca é uma característica da banana, que apresenta teores de potássio em casaca superiores a diversas outras frutas convencionais (Gondim et al., 2005). O teor de cálcio encontrado na farinha de casca deste estudo (Tabela 1), não corrobora outras pesquisas, onde o cálcio apresentou teores inferiores apenas ao do potássio (Cândido, 2020; Eshak, 2016; Gondim et al., 2005).

Os altos valores de potássio apresentados em ambas as farinhas refletem uma característica da fruta (Dotto et al., 2019), visto que em diferentes cultivares de banana, o potássio é o elemento extraído e exportado em maior quantidade. Assim como, os altos valores de manganês (Tabela 1), refletem as necessidades da cultura, visto que o Mn é o micromineral (ou 
micronutriente para termo agronômico) extraído e exportado em maiores quantidades por bananeiras. O teor de fósforo ficou abaixo apenas do potássio em ambas as farinhas (casca e polpa) (Tabela 1), o que pode ser devido ao alto teor de magnésio apresentado por elas (Tabela 1), pois a absorção e o transporte de fósforo estão ligados a um maior teor de magnésio na planta. Por outro lado, o teor mais modesto de enxofre reflete as baixas exigências agronômicas da cultura para esse elemento (Borges et al., 2016).

O maior teor de magnésio na farinha de casca em relação a farinha de polpa (Tabela 1) pode ser devido a presença de clorofila, molécula que tem o magnésio como um dos seus constituintes (Taiz et al., 2017) e que está presente nas cascas de bananas verdes (Reginio Jr. et al., 2020). Os maiores teores de potássio e sódio nas farinhas de cascas (Tabela 1) podem estar relacionados a atuação osmótica desses íons (Taiz et al., 2017), justificado pela maior umidade presente na casca do que na polpa de frutos verdes (Cândido, 2020) e, no caso do potássio, pela presença de estômatos nas cascas dos frutos (Banks et al., 1993), onde o nutriente atua nos movimentos de abertura e fechamento (Taiz et al., 2017). O fósforo presente em maior quantidade na farinha de casca (Tabela 1) pode estar relacionado ao maior teor de lipídeos dessa farinha (Cândido, 2020), de modo que, parte dessa fração pode ser composta por fosfolipídios presentes em membranas celulares, principalmente nos tilacoides (Taiz et al., 2017).

Tabela 1. Minerais em farinhas de polpa e casca de banana vermelha 'São Domingos' (base úmida).

\begin{tabular}{|c|c|c|c|c|c|c|c|c|c|c|c|}
\hline & $\mathbf{P}$ & $\mathbf{K}$ & $\mathbf{C a}$ & Mg & $\mathbf{S}$ & $\mathbf{F e}$ & $\mathrm{Cu}$ & Zn & Mn & $\mathbf{N a}$ & \multirow{2}{*}{ C.V\% } \\
\hline & & \multicolumn{4}{|c|}{$\mathrm{g} \mathrm{kg}^{-1}$} & \multicolumn{5}{|c|}{$\mathrm{mg} \mathrm{kg}^{-1}$} & \\
\hline Casca & $1,35 * \mathrm{~B}$ & $31,5^{*} \mathrm{~A}$ & $0,71 \mathrm{C}$ & $1,53 * \mathrm{~B}$ & $0,65 \mathrm{C}$ & $8,93 b$ & $3,47 \mathrm{c}$ & $17,5 * b$ & $27,5 \mathrm{a}$ & $11,8 * b$ & $10 ; 14$ \\
\hline Polpa & $0,64 \mathrm{~B}$ & $7,88 \mathrm{~A}$ & $0,37 \mathrm{C}$ & $0,67 \mathrm{~B}$ & $0,54 \mathrm{~B}$ & $6,67 b$ & $3,60 \mathrm{~b}$ & $3,47 b$ & $15,5 \mathrm{a}$ & $7,0 \mathrm{~b}$ & $5 ; 17$ \\
\hline C.V\% & 12,6 & 13,9 & 29,1 & 22,4 & 9,7 & 59,9 & 21,7 & 14,9 & 24,6 & 19,5 & \\
\hline
\end{tabular}

Valores com '*' na mesma coluna diferem estatisticamente entre si pelo teste $\mathrm{F}(p<0,05)$. Letras diferentes na mesma linha (maiúsculas para macrominerais e minúsculas para microminerais) diferem estatisticamente pelo teste Scott-Knott $(p<0,05)$. Fonte: Autores.

Apesar dos teores apresentados na tabela acima (Tabela 1), é importante deixar claro que, devido a metodologia utilizada, os valores representam os teores totais dos respectivos minerais. Assim, não significa que serão assimilados pelo organismo após a ingestão, visto que muitas interações entre os minerais e outros componentes dos alimentos podem interferir em sua biodisponibilidade (Anyasi et al., 2018; Gharibzahedi \& Jafari, 2017). No entanto, pode ser útil para rotulação do percentual da Ingestão Diária Recomendada de minerais na embalagem (Resolução da Diretoria Colegiada [RDC] n. 360, 2003).

A farinha de polpa apresentou maior teor de amido resistente (Tabela 2), um reflexo do maior teor de amido presente na farinha polpa em relação a farinha de casca (Cândido, 2020). Outras farinhas de polpa de triploides AAA apresentaram

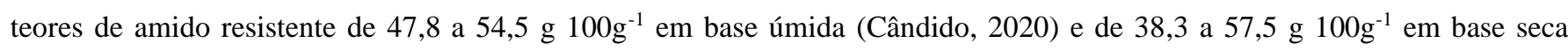
(Campuzano et al. 2018; Sardá et al., 2016). A diferença entre os teores de amido resistente presentes em farinhas de casca e de

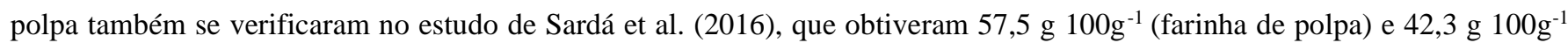
(farinha de casca).

Em um estudo que comparou 12 farinhas de polpa de banana obtidas em mercado, Sardá et al. (2016) encontraram

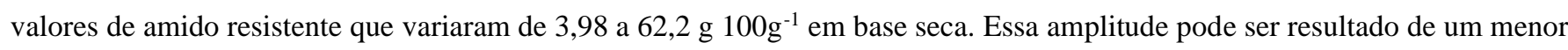
rigor na escolha dos frutos para a produção das farinhas, visto que, mesmo chamados de verdes, a diferença de amido entre os estádios 1 e 3 (Von Loesecke, 1950) pode ser de até 70\% menor no terceiro estádio de maturação em bananas triploides AAA (Campuzano et al. 2018). 
Tabela 2. Amido resistente em farinhas de polpa e casca de banana vermelha 'São Domingos'.

\begin{tabular}{ccc}
\hline & Base úmida $\left(\mathrm{g} 100 \mathrm{~g}^{-1}\right)$ & Base seca $\left(\mathrm{g} 100 \mathrm{~g}^{-1}\right)$ \\
\hline Casca & 27,7 & 28,9 \\
Polpa & $52,3^{*}$ & $54,7^{*}$ \\
\hline C.V \% & 7,3 & 7,4 \\
\hline
\end{tabular}

Valores com '*' na mesma coluna diferem estatisticamente entre si pelo teste $\mathrm{F}(p<0,05)$. Fonte: Autores.

\section{Conclusão}

O teor de umidade não diferiu entre as farinhas. A farinha de casca apresentou maiores teores de: fósforo, potássio, magnésio, zinco e sódio. O potássio e o manganês foram, respectivamente, o macro e o micromineral presentes em maiores quantidades em ambas as farinhas. No geral, o teor de minerais (cinzas) presentes na farinha de casca é superior à 300\% àquele presente na farinha de polpa. Por outro lado, a farinha de polpa apresenta quase $90 \%$ a mais de amido resistente que a farinha de casca, o que a torna uma farinha de característica desejável para aplicação industrial. Assim, as farinhas de casca e polpa obtidas a partir da variedade de casca vermelha 'São Domingos' (AAA) apresentam características de minerais, amido resistente e umidade semelhantes às de outras variedades triploides AAA.

Diferentemente das variedades de banana de casca amarela, as bananas de cascas vermelhas ainda não possuem um consenso em relação a sua maturação. Sabe-se que a maturação interfere nos teores de minerais e amidos. Assim, pesquisas futuras para caracterizar os estádios de maturação da banana 'São Domingos' e correlacioná-los com seus teores de amidos e minerais, ajudarão a identificar o ponto de maturação dos frutos para o preparo de farinhas sem perdas significativas desses nutrientes.

\section{Referências}

Al-Sahlany, S. T. G. \& Al-Musafer, M. A. S. (2020). Effect of substitution percentage of banana peels flour in chemical composition, rheological characteristics of wheat flour and the viability of yeast during dough time. Journal of the Saudi Society of Agricultural Sciences, 19(1), 87-91. https://doi.org/10.1016/j.jssas.2018.06.005

Alcântara, R. G., Fukumasu, H., Raspantini, P. C. F., Raspantini, L. E. R., Steel, C. J., Oliveira, L. C., Carvalho, R. A. \& Vanin, F. M. Baking effect on resistant starch digestion from composite bread produced with partial wheat flour substitution. (2020). Journal of Food Quality, 20. https://doi.org/10.1155/2020/9245035

American Association of Cereal Chemists. (1983). Approved methods of the American Association of Cereal Chemists, 1.

Amorim, E. P., Santos-Serejo, J. A., Amorim, V. B. O. \& Oliveira e Silva, S. (2016). Melhoramento genético. In C. F. Ferreira; S. Oliveira e Silva; E. P. Amorim \& J. A. Santos-Serejo (Ed.). O agronegócio da banana (pp 171-200). Embrapa.

Anyasi, T. A., Jideani, A. I. O. \& Mchau, G. R. A. (2018). Phenolics and essential mineral profile of organic acid pretreated unripe banana flour. Food Research International, 104, 100-109. https://doi.org/10.1016/j.foodres.2017.09.063

Banks, N. H., Dadzie, B. K. \& Cleland, D. J. (1993). Reducing gas exchange of fruits with surface coatings. Postharvest Biology and Technology, 3(3), 269284. https://doi.org/10.1016/0925-5214(93)90062-8

Borges, A. L., Silva, J. T. A., Oliveira, A. M. G. \& D’Oliveira, P. S. Nutrição e adubação. In C. F. Ferreira; S. Oliveira e Silva; E. P. Amorim \& J. A. SantosSerejo (Ed.). O agronegócio da banana (pp 331-398). Embrapa.

Borges, A. M., Pereira, J. \& Lucena, E. M. P. (2009). Caracterização da farinha de banana verde. Ciência e Tecnologia de Alimentos, 29(2), 333-339. https://doi.org/10.1590/S0101-20612009000200015

Cândido, H. T. (2020). Avaliação do sistema de produção de banana no município de Botucatu-SP: caracterização da maturação dos frutos e das farinhas de banana verde [Dissertação de Mestrado, Universidade Estadual Paulista (Unesp), Faculdade de Ciências Agronômicas]. Repositório Institucional Unesp. https://repositorio.unesp.br/handle/11449/192362

Campuzano, A., Rosell, C. M. \& Cornejo, F. (2018). Physicochemical and nutritional characteristics of banana flour during ripening. Food Chemistry, 256(1), 11-17. https://doi.org/10.1016/j.foodchem.2018.02.113

Cozzolino, S. M. F. (1997). Biodisponibilidade de minerais. Revista de Nutrição, 10(2), 87-98. https://doi.org/10.1590/S1415-52731997000200001

Cozzolino, S. M. F. (2007). Deficiências de minerais. Estudos Avançados, 21(60), 119-126. https://doi.org/10.1590/S0103-40142007000200009 
Dotto, J.; Matemu, A. O. \& Ndakidemi, P. A. Nutrient composition and selected physicochemical properties of fifteen Mchare cooking bananas: A study conducted in northern Tanzania. Scientific African, 6, 2019. https://doi.org/10.1016/j.sciaf.2019.e00150

Eshak, N. S. (2016). Sensory evaluation and nutritional value of balady flat bread supplemented with banana peels as a natural source of dietary fiber. Annals of Agricultural Science, 61(2), 229-235. https://doi.org/10.1016/j.aoas.2016.07.002

Falcomer, A. L., Riquette, R. F. R., Lima, B. R.; Ginani, V. C. \& Zandonadi, R. P. (2019). Health Benefits of green banana consumption: a systematic review. Nutrients, 11(6), 1222. https://doi.org/10.3390/nu11061222

Food and Agriculture Organization of the United Nations. FAOSTAT: Crop. http://www.fao.org/faostat/en/\#data/QC

Ferreira, D. F. (2014). Sisvar: a guide for its Bootstrap procedures in multiple comparisons. Ciência e Agrotecnologia, 38(2), 109-112. https://doi.org/10.1590/S1413-70542014000200001

Fu, X., Cheng, S., Liao, Y., Huang, B.; Du, B., Zeng, W., Jiang, Y., Duan, X. \& Yang, Z. (2018). Comparative analysis of pigments in red and yellow banana fruit. Food Chemistry, 239, 1009-1018. DOI: 10.1016/j.foodchem.2017.07.046

Gharibzahedi, S. M. T. \& Jafari, S. M. (2017). The importance of minerals in human nutrition: bioavailability, food fortification, processing effects and nanoencapsulation. Trends in Food Science \& Technology, 62, 119-132. https://doi.org/10.1016/j.tifs.2017.02.017

Gondim, J. A. M., Moura, M. F. V., Dantas, A. S.; Medeiros, R. L. S. \& Santos, K. M. (2005). Composição centesimal e de minerais em cascas de frutas. Ciência e Tecnologia de Alimentos, 25(4), 825-827. https://doi.org/10.1590/S0101-20612005000400032

Goñi, I.; Garcia-Diz, L.; Mañas, E., \& Saura-Calixto, F. (1996). Analysis of resistant starch: a method for foods and food products. Food Chemistry, 56(4), 445-449. https://doi.org/10.1016/0308-8146(95)00222-7

Instituto Brasileiro de Geografia e Estatística. (2021). Censo Agro 2017: Resultados definitivos. https://censos.ibge.gov.br/agro/2017/templates/censo_agro/resultadosagro/agricultura.html?localidade=0\&tema=76237

Khoozani, A. A., Kebede, B., Birch, J. \& Bekhit, A. E. A. (2020). The effect of bread fortification with whole green banana flour on its physicochemical, nutritional and in vitro digestibility. Foods, 20(2), 152. https://doi.org/10.3390/foods9020152

Kumar, P. S., Saravanan, A.; Sheeba, N. \& Uma, S. (2019). Structural, functional characterization and physicochemical properties of green banana flour from dessert and plantain bananas (Musa spp.). LWT - Food Science and Technology, 116. https://doi.org/10.1016/j.lwt.2019.108524

Leão, A. L. M. \& Santos, L. C. (2012). Consumo de micronutrientes e excesso de peso: existe relação? Revista Brasileira de Epidemiologia, 15(1), 85-95. http://dx.doi.org/10.1590/S1415-790X2012000100008

Lekshmi, P. R. G., Philipose, J. \& Nair, C. S. J. (2019). Development of grading system in Red Banana for value addition. Environment and Ecology, 37(1), 281-286. http://www.environmentandecology.com/

Lokesh, V.; Divya, P.; Puthusseri, B.; Manjunatha, G. \& Neelwarne, B. (2014). Profiles of carotenoids during post-climacteric ripening of some important cultivars of banana and development of a dry product from a high carotenoid yielding variety. LWT - Food Science and Technology, 55(1), 59-66. https://doi.org/10.1016/j.1wt.2013.09.005

Lorenzi, H., Lacerda, M. T. C. \& Bacher, L. B. (2015). Frutas no Brasil nativas e exóticas: (de consumo in natura). Instituto Plantarum de Estudos da Flora.

Marles, R. J. (2017). Mineral nutrient composition of vegetables, fruits and grains: the context of reports of apparent historical declines. Journal of Food Composition and Analysis, 56, 93-103. https://doi.org/10.1016/j.jfca.2016.11.012

Reginio Jr., F. C.; Ketnawa, S. \& Ogawa, Y. (2020). In vitro examination of starch digestibility of Saba banana [Musa 'saba' (Musa acuminata $\times$ Musa balbisiana)]: impact of maturity and physical properties of digesta. Scientific Reports, 10, 1811. https://doi.org/10.1038/s41598-020-58611-5

Resolução da Diretoria Colegiada - RDC no 263, de 22 de setembro de 2005. (2005, 23 setembro). Dispõe sobre o Regulamento Técnico para produtos de cereais, amidos, farinhas e farelos". Diário Oficial [da] República Federativa do Brasil.

Resolução da Diretoria Colegiada - RDC nº 360, de 23 de dezembro de 2003. (2003, 23 dezembro). Aprova regulamento técnico sobre rotulagem nutricional de alimentos embalados, tornando obrigatória a rotulagem nutricional. Diário Oficial [da] República Federativa do Brasil.

Sardá, F. A. H., Lima, F. N. R., Lopes, N. T. T., Santos, A. O., Tobaruela, E. C., Kato, E. T. M. \& Menezes, E. W. (2016). Identification of carbohydrate parameters in commercial unripe banana flour. Food Research International, 81, 203-209. https://doi.org/10.1016/j.foodres.2015.11.016

Silva, A. A., Barbosa Junior, J. L. \& Barbosa, M. I. M. J. (2015). Farinha de banana verde como ingrediente funcional em produtos alimentícios. Ciência Rural, 45(12), 2252-2258. http://dx.doi.org/10.1590/0103-8478cr20140332

Souza, A. P. S., Cândido, H. T., Santos, T. P. R. \& Leonel, M. (2019). Caracterização da maturação da banana 'São Domingos'. Anais do Simpósio Nacional de Tecnologia em Agronegócio, 11(1), 123-132.

Suntharalingam, S. \& Ravindran, G. (1993). Physical and biochemical properties of green banana flour. Plant Foods for Human Nutrition, 43(1), 19-27. DOI: 10.1007/BF01088092

Taiz, L. [et al.]. (2017). Fisiologia e desenvolvimento vegetal (6nd Ed). (A. A. Mastroberti, et al., Trad.). Artmed.

Valença, A. W., Bake, A., Brouwer, I. D. \& Giller, K. E. (2017). Agronomic biofortification of crops to fight hidden hunger in sub-Saharan Africa. Global Food Security, 12, 8-14. https://doi.org/10.1016/j.gfs.2016.12.001 
Research, Society and Development, v. 10, n. 4, e1810413860, 2021

(CC BY 4.0) | ISSN 2525-3409 | DOI: http://dx.doi.org/10.33448/rsd-v10i4.13860

Viana, E. S., Souza, A. S., Reis, R. C. \& Oliveira, V. J. S. (2018). Application of green banana flour for partial substitution of wheat flour in sliced bread. Semina: Ciências Agrárias, 39(6), 2399-2408. http://dx.doi.org/10.5433/1679-0359.2018v39n6p2399

Vieira, S. Análise de variância: (anova). (2006). Atlas.

Von Loesecke, H. W. (1950). Bananas. 2 nd. Interscience Publisher. 